\title{
Applying Phenomenography to Develop a Comprehensive Understanding of Ethics in Engineering Practice
}

\author{
Andrew O. Brightman \\ Weldon School of Biomedical \\ Engineering \\ Purdue University \\ West Lafayette, IN, USA \\ aob@purdue.edu
}

\author{
Nicholas D. Fila \\ Electrical and Computer \\ Engineering \& Industrial Design \\ Iowa State University \\ Ames, IA, USA \\ nfila@iastate.edu
}

\author{
Justin L. Hess \\ STEM Education Innovation \& \\ Research Institute \\ IUPUI \\ Indianapolis, IN, USA \\ hessil@iupui.edu
}

\author{
Alison J. Kerr \\ Department of Psychology \\ University of Tulsa \\ Tulsa, OK, USA \\ ajk050@utulsa.edu
}

\author{
Dayoung Kim \\ School of Engineering Education \\ Purdue University \\ West Lafayette, IN, USA \\ kim2603@purdue.edu
}

\author{
Michael C. Loui \\ School of Engineering Education \\ Purdue University \\ West Lafayette, IN, USA \\ mloui@purdue.edu
}

\author{
Carla B. Zoltowski \\ School of Electrical and Computer \\ Engineering \\ Purdue University \\ West Lafayette, IN, USA \\ cbz@purdue.edu
}

\begin{abstract}
This Work-in-Progress Research paper describes (1) the contemporary research space on ethics education in engineering; (2) our long-term research plan; (3) the theoretical underpinnings of Phase 1 of our research plan (phenomenography); and (4) the design and developmental process of a phenomenographic interview protocol to explore engineers' experiences with ethics. Ethical behavior is a complex phenomenon that is complicated by the institutional and cultural contexts in which it occurs. Engineers also have varied roles and often work in a myriad of capacities that influence their experiences with and understanding of ethics in practice. We are using phenomenography, a qualitative research approach, to explore and categorize the ways engineers experience and understand ethical engineering practice. Specifically, phenomenography will allow us to systematically investigate the range and complexity of ways that engineers experience ethics in professional practice in the health products industry. Phenomenographic data will be obtained through a specialized type of semi-structured interview. Here we introduce the design of our interview protocol and its four sections: Background, Experience, Conceptual, and Summative. We also describe our iterative process for framing questions throughout each section.
\end{abstract}

Primary Topic: Engineering Education Research; Secondary Topics: Engineering Ethics; Professional Ethics; Qualitative Research Methodologies

\section{INTRODUCTION}

Recent news reports, such as the emissions scandal at Volkswagen and the water crisis in Flint, show how engineers can substantially affect a wide array of stakeholders in terms of health, public safety, and the environment. Such examples illustrate how engineers' decisions are influenced by factors such as corporate cultural norms, laws and regulations, and societal values. These factors combine to create challenging

This material is based upon work supported by the National Science Foundation under Grant No. 1737303. Any opinions, findings, and conclusions or recommendations expressed in this material are those of the author(s) and do not necessarily reflect the views of the National Science Foundation. environments in which engineers make ethical decisions they may not have imagined when they were preparing for their careers. Ethical concerns are particularly salient for those working in the highly competitive health products industry. For example, a recent high profile litigation case which involved the erosion of synthetic surgical implant devices left medical device manufacturers wondering, "How could this long-term pain and suffering of so many women have been prevented?" and "Could this 'black eye' on this health product industry have been avoided by mitigation of ethical issues up front or through better training of our engineering workforce?" [1]

Enhanced ethics education in engineering curricula is one viable mechanism for developing engineering practitioners who are adept at addressing ethical concerns. However, engineering education researchers have identified a lack of alignment between the complexities of lived engineering contexts and the ways that academia trains and evaluates the ethical abilities and dispositions of engineering students [2-4]. Aligning the practices of ethics education with the reality of engineering practice first requires an empirically grounded and context-specific understanding of the complex ways in which engineers experience ethics in their practice. While the need for richer and more contextually-specific ethics training exists for many areas, the potential for harm in the healthcare industry, both domestically [5-8] and internationally [9-11], makes it an ideal disciplinary space in which to focus this project.

\section{BACKGROUND}

Since ABET has required "an understanding of professional and ethical responsibility" as an outcome of all accredited engineering programs (EC2000), strategies for addressing ethics across engineering curricula have proliferated. Yet, identifying this outcome only as "an understanding" focused efforts largely on learning ethics 
codes and professional responsibility rather than on practicing applications of ethics based on real-world engineering experiences. The recently updated ABET outcomes now specify not only understanding but also judgment. As outcome 5 now states, students must have: "An ability to recognize ethical and professional responsibilities in engineering situations and make informed judgments, which must consider the impact of engineering solutions in global, economic, environmental, and societal contexts" [12].

Currently, professional codes are among the most common frameworks for teaching engineering ethics. Nearly all professional engineering societies and organizations have created ethics codes. While codes are important to understanding ethical practice in specific engineering disciplines and in engineering generally [13-15], students may not see the direct applicability of codes to everyday ethical practice [16]. Codes may also not address ethical issues that arise with emerging technologies [5].

In addition, engineering instructors have offered several theoretical frameworks and approaches focused on ethical reasoning and decision-making. Examples include reflexive principlism [3], an approach centered on macro-micro perspectives [17], and an approach focused on meta-ethics [18]. However, it is not clear that these approaches capture the full range of situated ethical engineering practice.

Social justice [19], empathy [20], and care [21] offer other frameworks for situating ethics in engineering curricula. Yet, as Swiestra and Jelsma [22] wrote, "Scanning the [Science, Technology, \& Society] literature on ethical aspects of engineering practice delivered no systematic data about typical ways in which practicing engineers think about their social responsibility" (p. 315). While some research has partially filled this gap [23, 24], these studies tend to explore the perceptions of students rather than the experiences of practitioners. Furthermore, approaches are often broadly situated and not context-specific.

\section{RESEARCH OVERVIEW}

Through a systematic qualitative research study, we aspire to develop a comprehensive understanding of ethical engineering practice. Our study will enable us to analyze the alignment of relationships among frameworks for ethics education in engineering and the reality of engineering practice within the health products industry. Our study is driven by three research questions:

RQ1: What are the qualitatively different ways engineers experience ethical issues in their engineering practice in the health products industry?

RQ2: What individual and environmental factors influence an engineer's experience of ethical engineering practice?

RQ3: To what extent are current frameworks of ethics education aligned with a comprehensive and situated understanding of ethical engineering practice?

Because phenomenography is a qualitative research methodology which explores the ways in which a phenomenon is experienced, it is an ideal method for examining the embedded values and the range of ways that engineers view and respond to ethical issues in their practice. Hence, to address RQ1, we will utilize phenomenography to develop a comprehensive framework, grounded in the lived experiences of engineers, that describes the phenomenon of ethical engineering in actual practice within the health products industry.

While phenomenographic interview and analysis methods will enable us to address RQ1 and to describe the variation in ways that engineers experience ethics, it will not necessarily identify which factors best contribute to the development of ethical engineering practitioners. Thus, to address RQ2, we also plan to utilize thematic and content analysis approaches to identify factors that influence the formation of a comprehensive understanding of ethical engineering practice.

As discussed in the Background section, there are many frameworks and approaches applied to training future engineers to become ethical practitioners. To further understand their applicability, based on the results generated in addressing RQs 1 and 2, we will address RQ3 by analyzing currently used frameworks and approaches. We will deduce the interrelationships and model their alignment with the findings from RQ1 and RQ2 to identify the appropriateness of existing strategies and (as applicable) the need for novel strategies to prepare future ethical practitioners.

\section{PHENOMENOGRAPHY}

Phenomenography enables researchers to develop a comprehensive understanding of how people experience a complex human activity, generally called a phenomenon. Phenomenography is a qualitative, empirical research methodology that arose from a recognition that the qualitatively different ways in which learners experience and understand a phenomenon are related to the qualitative differences in learning outcomes $[25,26]$. By exploring the variations along multiple dimensions, researchers can develop a conceptualization that honors the diverse range of ways individuals experience the phenomenon [27].

Phenomenography differs from phenomenology, which emphasizes understanding the essence of the lived experience of the phenomenon [28]. Phenomenography has been used widely in educational research [29] and has recently been applied to analyze challenging problems in engineering education [30], environmental education [31], computer science [32, 33], and professional practice [34-37]. This study is the first application of phenomenography to ethics that we know of.

The "unit of phenomenographic research is a way of experiencing something" [27]. This idea refers to the aspects of the phenomenon that one attends to when interacting with it in a specific setting. The results of phenomenographic research form an outcome space that contains (1) categories of description and (2) the structural relationships among the categories. Phenomenographic analysis produces a limited number of categories of description that represent a hierarchy from less to more comprehensive ways individuals understand the phenomenon. Thus, several categories might share similar 
aspects, but each subsequent category will entail more aspects (and thus a more complete framing of the phenomenon) than the less comprehensive categories. In this way, the structural relationships describe ways in which particular categories are more complete than others, often along particular dimensions of variation.

As one seminal example of phenomenography, Marton \& Booth [27] derived a two-dimensional outcome space of learning that differed in two facets: ways of experiencing learning and temporally. Ways of experiencing learning included committing words to memory, committing meaning to memory, and understanding meaning. The temporal facet differed in terms of acquiring, knowing, and making use of knowledge.

In an engineering-specific example of phenomenography, Zoltowski et al. [38] derived a two-dimensional outcome space of the variation in ways engineering students experience human-centered design. The categories of description ranged from "user input" to "linear process" to "empathic design" ( $p$. 41). Each category represented an increase in comprehensiveness of understanding along one or two dimensions: understanding of the users and design process and integration.

\section{Design of PhENOMENOGRAPHIC INTERVIEW Protocol}

In phenomenographic studies, interviews are the primary form of data collection. Interviews provide an effective means for researchers to enter participants' lives and explore their experiences of the phenomenon being studied [39]. A semistructured approach to the interviews allows the interviewer to guide the participants to reflect on their experiences with the phenomenon and ensure clarification, elaboration, and deeper reflection as necessary $[39,40]$.

The goal of a phenomenographic interview is to collect a complete and unbiased account of a participant's experiences with and understanding of the phenomenon. It is important to establish an interview context and protocol that allow the participant to (1) access their direct experience(s) with the phenomenon, (2) describe them in detail, and (3) avoid bias by statements from the interviewer. Hence, interviews are structured in this way to build from situated examples, which enable the participant to holistically describe their way of experiencing a phenomenon. These examples allow the participant to articulate abstracted conceptualizations, or what they understand to be the key features of their way of experiencing the phenomenon $[27,39,40]$. Further, since the variation-seeking aspects of phenomenography emphasize comparison between and across participants, all participants should have equal opportunities to access and discuss their experiences and conceptualizations.

Because the goal of phenomenography is to capture variety and breadth of the experience of a phenomenon, our team developed a pre-interview screening survey to select participants. The aim of this survey was to ensure that we obtain a comprehensive representation of relevant demographics across both engineering practitioners and interns to capture the broadest range of experiences possible. The following sections describe the development of both the screening survey and the phenomenographic interview questions and protocol.

Our interview protocol was built on a structure common to phenomenographic interviews [38, 41] and included four sections: (1) Background, (2) Experience with Phenomenon, (3) Conceptual Understanding, and (4) Summative Reflection. Our team originally designed the interview protocol from those we had previously used [38, 42]. However, these previous protocols could not be adopted verbatim because they differed in two key ways. First, the previous phenomena were innovation and human-centered design rather than ethics. Second, the participants were students rather than practitioners.

After creating a draft protocol, we took four steps to support the final design of our protocol: (1) expert review, (2) pilot interviews with debriefing sessions, (3) interviewer review of transcripts and reflection, and (4) team discussion and iteration based on these activities. Our team developed several drafts of both the screening survey and the interview protocol. Expert review of the survey and pilot interviews led us to realize that both sets of questions required an extensive amount of time. Team discussions focused on refinements and streamlining, while still achieving the objectives of the survey and the interview. In the following sections, we describe each of the interview components, iterations made, and example questions.

\section{A. Screening Survey}

In the screening survey, we collected background information to identify and select participants for interviews. This information included level of education, academic discipline, age, gender, race, and nationality. Additionally, we collected basic aspects of current engineering practice, such as whether the engineer is currently an intern or practitioner, the number of years they have worked in engineering, their field, and their current role. Early survey versions included more questions about ethical experiences, but since those experiences were the intended focus of the phenomenographic interviews, we retained only two questions on ethics experiences. One survey question asked respondents to identify the types of ethics training they have had, such as college coursework, workplace training, workshops, or independent reading. The other question asked respondents to report the frequency with which they perceived ethical issues to arise in their work. Thus, the screening survey collected initial background information to ensure that interview participants would be selected with a wide range of professional experiences and ethics training.

\section{B. Background Section}

The Background section of a phenomenographic interview captures the participant's educational, professional, and life experiences, which inform their experience and understanding of the phenomenon. Discussing educational backgrounds, professional roles, and life experiences can help build rapport with participants, contextualize the experiential responses, and inform follow-up questions. Hence, some background information questions from the survey (with a few additions) were incorporated into the interview protocol. Additional 
questions about educational pursuits and professional experiences are asked as opening interview questions. Examples of retained background interview questions are, "I see that you that you are working in [specify field], and that you have worked for [specify duration] in that field. What has that been like? How did you decide to work in that field?"

\section{Experience with Phenomenon}

The Experience with Phenomenon section of the interview focuses on uncovering a thorough account of one or more key encounters the participant has had with the phenomenon, including their understanding of these encounters. Typically, the experiential phase begins by asking the participant to describe an experience they have had with the phenomenon. Due to piloting and internal discussion, we made three substantive changes to the experiential section. Specifically, we (1) revised the phrasing of the phenomenon as communicated during the interview, (2) altered the wording, structure, and inclusion of follow-up questions, and (3) we generated heuristics to discern when an experience was described in sufficient detail.

\section{1) Phrasing of Phenomenon}

The phrasing of the phenomenon establishes the focal point for the remainder of the interview and was a key point of discussion and iteration. Initially, we prompted participants to describe an experience they have had with ethics at work or ethical engineering practice. After several revisions, our team established this phrasing as follows: "Can you describe an experience you have had with ethics in engineering?" This revision provided a clearer focus for participants to describe their experiences with the phenomenon.

\section{2) Follow-up Questions}

Originally, we had listed 15 follow-up questions that the interviewers could use based on the flow of conversation. This list was revised, whereby questions were prioritized and the list was shortened to seven questions. In addition, we added a separate sub-section on "Clarifications and Expansion." For example, a clarification question could include, "Would you explain what you mean by..."

\section{3) Emphasizing Depth over Breadth of Experiences}

We initially planned for interviews to last approximately 90 minutes. In one pilot interview, however, the participant discussed three experiences for 2.5 hours. Given the need to explore a phenomenon thoroughly, our team has established a set of heuristics to determine when appropriate depth of description has been achieved.

\section{Conceptual Understanding}

The Conceptual Understanding section elicits the participant's abstract and theoretical understandings of the phenomenon. The experiences previously discussed provided a grounding for these explanations. We removed several questions, especially those that had the potential to introduce concepts that participants had not previously discussed. However, we retained questions such as, "What experiences do you believe contributed the most to your understanding of ethics in engineering?" as questions such as this are relevant to the thematic analysis portion of the study (RQ2).

\section{E. Summative Reflection}

This section allows the participant to reflect (one last time) on their experience of the phenomenon and clarify, refine, or add to any of their previous responses. Questions prompt participants to consider if there is anything they did not mention but wished to. One closing question in this section is, "How accurately and thoroughly do you think you communicated your experiences and perspectives?"

\section{DELIVERY OF PROTOCOL}

One point of emphasis among our team was developing consistency across interviewers. Often, in phenomenographic studies there is one interviewer. However, in our case we are dividing interviews among three individuals.

We conducted a critical review of the transcripts of three pilot interviews, each conducted by one of the three interviewers. This review allowed each interviewer to reflect on their own interviewing style, with its merits and weaknesses. In addition, this review allowed for a calibration of interviewing techniques and strategies across interviewers.

As one example of a suggested improvement in technique, after review of a pilot interview that he conducted, an interviewer noticed that he rarely asked "why," "feel," and "connect" questions. In addition, a peer interviewer prompted him to devote more time to exploring a depth of experience rather than breadth of several experiences. For example, follow-up questions like, "Tell me more about..." can and should be utilized to continue a conversation on an aspect of an experience rather than quickly transitioning to another aspect or altogether new experience.

\section{CONCLUSION}

This study outlined the need for a comprehensive understanding of ethical engineering practice that is grounded in the lived reality of engineering practitioners. We also outlined the methodology our team will use to address this need, phenomenography. There are many strategies for training ethical engineers, but without exploring the reality of ethical issues encountered in daily practice, these educational strategies run the risk of being irrelevant and ineffective for training engineers to respond to ethical issues in their careers. We argued that phenomenography was the ideal method for uncovering the range in ways of experiencing ethics in engineering practice. The effectiveness of this method is largely contingent upon a valid and unbiased extraction of experiences. This is especially important as we seek to uncover variations in ethics, which can be a particularly sensitive and nuanced topic for research. We hope that this study will serve as a guidepost for effectively training future ethical practitioners. 


\section{REFERENCES}

[1] A. O. Brightman, M. Hiles, and J. Beever, "Next generation ethical development of medical devices: Considering harms, benefits, fairness, and freedom," in Next Generation Ethics: Engineering a Better Society, A. E. Abbas and S. Gee, Eds., 2018.

[2] B. E. Barry and M. W. Ohland, "Applied ethics in the engineering, health, business, and law professions: A comparison," Journal of Engineering Education, vol. 98, no. 4, pp. 377-388, 2009.

[3] J. Beever and A. O. Brightman, "Reflexive Principlism as an effective approach for developing ethical reasoning in engineering," Science and Engineering Ethics, vol. 22, no. 1, pp. 275-291, 2016.

[4] D. Bairaktarova and A. Woodcock, "Engineering student's ethical awareness and behavior: A new motivational model," Science and Engineering Ethics, vol. 23, no. 4, pp. 1129-1157, 2017.

[5] M. Kessel, "Restoring the pharmaceutical industry's reputation," Nature Biotechnology, vol. 32, no. 10, pp. 983-990, 2014

[6] J. Levitz and J. Kamp, Deadly medicine: A common surgery for women and the cancer it leaves behind. The Wall Street Journal, 2017.

[7] B. J. Maron and R. G. Hauser, "Perspectives on the failure of pharmaceutical and medical device industries to fully protect public health interests," The American Journal of Cardiology, vol. 100, no. 1, pp. 147-151, 2007.

[8] E. J. Topol, "Failing the public health - Rofecoxib, Merck, and the FDA.," New England Journal of Medicine, vol. 351, no. 17, pp. $1707-$ 1709, 2004.

[9] R. Francis, Report of the Mid Staffordshire NHS Foundation Trust public inquiry. London, UK: The Stationery Office, 2013.

[10] M. A. Sujan, I. Habli, T. P. Kelly, A. Gühnemann, S. Pozzi, and C. W. Johnson, "How can health care organisations make and justify decisions about risk reduction? Lessons from a cross-industry review and a health care stakeholder consensus development process," Reliability Engineering \& System Safety, vol. 161, pp. 1-11, 5// 2017.

[11] K. Walshe and N. Offen, "A very public failure: lessons for quality improvement in healthcare organisations from the Bristol Royal Infirmary," Quality in Health Care, vol. 10, no. 4, pp. 250-256, 2001.

[12] ABET. (n.d.). Rationale for revising criteria 3. Available: http://www.abet.org/accreditation/accreditation-criteria/accreditationalerts/rationale-for-revising-criteria-3/

[13] C. E. Harris, M. Davis, M. S. Pritchard, and M. J. Rabins, "Engineering ethics: what? why? how? and when?," Journal of Engineering Education, vol. 85, pp. 93-96, 1996.

[14] C. E. Harris, "Internationalizing professional codes in engineering," Science \& Engineering Ethics, Article vol. 10, no. 3, pp. 503-521, 2004.

[15] National Society of Professional Engineers. (2013, February 15, 2015). NSPE Code of Ethics for Engineers. Available: http://www.nspe.org/Ethics/CodeofEthics/index.html

[16] W. T. Lynch and R. Kline, "Engineering practice and engineering ethics," Science, Technology \& Human Values, vol. 25, no. 2, pp. 195 225,2000

[17] J. R. Herkert, "Ways of thinking about and teaching ethical problem solving: Microethics and macroethics in engineering," Science and Engineering Ethics, vol. 11, pp. 373-385, 2005.

[18] D. R. Haws, "The importance of meta-ethics in engineering education," Science and Engineering Ethics, vol. 10, no. 2, pp. 204 210, 2004

[19] D. M. Riley and Y. Lambrinidou, "Canons against cannons? Social justice and the engineering ethics imaginary," presented at the American Society for Engineering Education, Seattle, WA, 2015.

[20] J. L. Hess, J. Beever, J. Strobel, and A. O. Brightman, "Empathic perspective-taking and ethical decision-making in engineering ethics education," in Philosophy and Engineering: Exploring Boundaries, Expanding Connections, D. Michelfelder, B. Newberry, and Q. Zhu, Eds.: Springer, 2017, pp. 163-179.

[21] M. Pantazidou and I. Nair, "Ethic of care: Guiding principles for engineering teaching \& practice," Journal of Engineering Education, vol. 88 , no. 2 , pp. 205-212, 1999.

[22] T. Swiestra and J. Jelsma, "Responsibility without moralism in technoscientific design practice," Science, Technology \& Human Values, vol. 31, no. 3, pp. 309-332, 2006.
[23] D. Nieusma and D. Riley, "Designs on development: Engineering, globalization, and social justice," Engineering Studies, vol. 2, no. 1, pp. 29-59, 2010

[24] N. Canney and A. Bielefeldt, "A framework for the development of social responsibility in engineers," International Journal of Engineering Education, vol. 31, no. 1, pp. 414-424, 2015.

[25] F. Marton, "Phenomenography: A research approach to investigating different understandings of reality," Journal of Thought, vol. 21, no. 3, pp. 28-49, 1986

[26] S. Booth, "On phenomenography, learning and teaching," Higher Education Research \& Development, vol. 16, no. 2, pp. 135-158, 1997.

[27] F. Marton and S. Booth, Learning and awareness. NJ: Lawrence Erlbaum Associates, 1997.

[28] A. Barnard, H. McCosker, and R. Gerber, "Phenomenography: A qualitative research approach for exploring understanding in health care," Qualitative Health Research, vol. 9, no. 2, pp. 212-226, 1999.

[29] M. Tight, "Phenomenography: The development and application of an innovative research design in higher education research," International Journal of Social Research Methodology, vol. 19, no. 3, pp. 319-338, 2016

[30] J. M. Case and G. Light, "Emerging methodoloiges in engineering education research," Journal of Engineering Education, vol. 100, no. 1, pp. 186-210, 2011

[31] T. Loughland, A. Reid, and P. Petocz, "Young people's conceptions of environment: A phenomenographic analysis," Environmental Education Research, vol. 8, no. 2, pp. 187-197, 2002.

[32] C. Bruce, L. Buckingham, J. Hynd, C. McMahon, M. Roggenkamp, and I. Stoodley, "Ways of experiencing the act of learning to program A phenomenographic study of introductory programming students at university," in Transforming IT Education: Promoting a Culture of Excellence, C. Bruce, G. Mohay, G. Smith, I. Stoodley, and R. Tweedale, Eds. Santa Rosa, CA: Informing Science Press, 2006, pp. 301-325.

[33] P. Kinnunen and B. Simon, "Phenomenography and grounded theory as research methods in computing education research field," Computer Science Education, vol. 22, no. 2, pp. 199-218, 2012.

[34] M. Forster, "Information literacy as a facilitator of ethical practice in the professions," Journal of Information Literacy, vol. 7, no. 1, pp. 1829, 2013

[35] V. Sappa and C. Aprea, "Conceptions of connectivity: How Swiss teachers, trainers and apprentices perceive vocational learning and teaching across different learning sites," Vocations and Learning, vol. 7, no. 3, pp. 263-287, 2014.

[36] M. Bailey, "Professional development of HR practitioners: A phenomenographic study," European Journal of Training and Development, vol. 39, no. 3, pp. 220-238, 2015.

[37] J. Brunstein, S. Bertossi Heidrich, and R. de Araújo Amaro, "Competencies for a fair play in organizations: A phenomenographic analysis of managers' conceptions," BBR-Brazilian Review, vol. 13, no. 2, pp. 105-134, 2016.

[38] C. B. Zoltowski, W. C. Oakes, and M. E. Cardella, "Students' ways of experiencing human-centered design," Journal of Engineering Education, vol. 101, no. 1, pp. 28-59, 2012.

[39] P. Ashworth and U. Lucas, "Achieving empathy and engagement: A practical approach to the design, conduct, and reporting of phenomenographic research," Studies in Higher Education, vol. 25, no. 3, pp. 295-308, 2000.

[40] G. Åkerlind, "Learning about phenomenography: Interviewing, data analysis and the qualitative research paradigm," in Doing developmental phenomenography, J. A. Bowden and P. Green, Eds. Melbourne, Australia: RMIT University Press, 2005.

[41] G. Åkerlind, "Phenomenographic methods: A case illustration," in Doing developmental phenomenography, , J. A. Bowden and P. Green, Eds. Melbourne, Australia: RMIT University Press, 2005.

[42] N. D. Fila, "The qualitatively different ways engineering students experience innovation during engineering design projects," $\mathrm{PhD}$, Purdue University, 2016. 